\title{
Centralized Rate Control Mechanism for Cellular-Based Vehicular Networks
}

\author{
Shiqiang Wang*, Long Le ${ }^{\dagger}$, Nikola Zahariev ${ }^{\dagger}$, and Kin K. Leung* \\ ${ }^{*}$ Department of Electrical and Electronic Engineering, Imperial College London, SW7 2AZ, United Kingdom \\ ${ }^{\dagger}$ NEC Laboratories Europe, NEC Europe Ltd., 69115 Heidelberg, Germany \\ Email: shiqiang.wang11@imperial.ac.uk, Long.Le@neclab.eu, Nikola.Zahariev@neclab.eu, kin.leung@imperial.ac.uk
}

\begin{abstract}
Vehicular networking requires high message transmission rate but also faces limited radio resources. This may lead to congestion in the radio access network and incur long delay for the messages. On the other hand, applications also have stringent requirement for latency (or freshness) of the received information. In this paper, we focus on cellular-based vehicular networks and propose a method for finding an optimal trade-off between network congestion and the freshness of received information. We suggest a feedback-based scheme for vehicles and a centralized entity (GeoServer) to coordinate with each other to determine a message transmission rate that best satisfies the application requirements. We first outline the framework of the proposed mechanism, and then analytically derive the optimal solution. Following that, the performance of the proposed mechanism is evaluated via simulations.
\end{abstract}

Index Terms-Cellular networks, congestion control, queuing theory, rate control, vehicular networks.

\section{INTRODUCTION}

Vehicular networking is considered as an important technology in the area of intelligent transportation systems (ITS) [1]. In the last decade, a vast amount of research has been done on vehicular networking based on WiFi-based multi-hop networks, where vehicles are equipped with WiFi communication devices to communicate with each other. A drawback of vehicular networking based on multi-hop networks is that a large percentage of vehicles must be equipped with such devices in order to make it work. Hence, in recent years, attention has been turned to how to leverage cellular networks such as 4th-Generation/Long-Term-Evolution (4G/LTE) to support vehicular networking [2], [3], which can be easily introduced to the market as a smartphone application.

A design approach for cellular-based vehicular networking is to introduce a new network element that serves as a message reflector to facilitate the communication among vehicles. This network element functions as a server. It processes incoming messages from each vehicle, aggregates these messages when appropriate, and redistributes the messages to other vehicles. Since this server is typically responsible for a geographical area, it is termed GeoServer [4], [5]. The main functionality of a GeoServer is to provide vehicles with geographical-related services such as safety- and commercial-related services. The application scenario is illustrated in Fig. 1.

An important application message in vehicular networking is the application-level beacon message, which is also called heartbeat, basic safety message, or cooperative awareness message (CAM) [6]. This message is periodically sent by

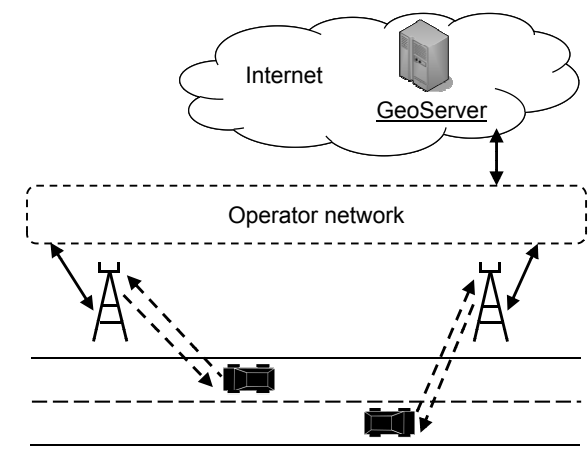

Fig. 1. Application Scenario.

each vehicle to the GeoServer ${ }^{1}$ and contains the vehicle's status such as position, speed, and heading. Using the periodic CAMs received from vehicles, the GeoServer can detect events such as potential collisions with other vehicles in its vicinity. Another important application message is the decentralized environmental notification message (DENM) [8], which the GeoServer periodically sends to vehicles. This message can include danger warnings, road instructions, etc.

Both CAMs and DENMs need to be transmitted in short intervals [1], [6], [8], but the radio resources are limited. This may lead to congestion in the radio access network and incur long delay for the messages. On the other hand, applications also have stringent requirement for latency (or freshness) of the received information. These two conflicting requirements pose a difficult problem for applications of vehicular networking. It is shown in [7] that standard message transmission rates may lead to large delay when communicating via the LTE network. However, no solution has been proposed until now, to the best of our knowledge. To address this issue, we propose a mechanism to adjust the message transmission rate for cellular-based vehicular networks in this paper. We first present a general framework of the proposed rate control mechanism, and then discuss the method of obtaining the optimal message transmission rate based on network observations. The goal of the proposed scheme is to maintain the received information

\footnotetext{
${ }^{1}$ Note that this setting is slightly different from multi-hop vehicular networks where CAMs are broadcasted to all neighboring vehicles, because in the cellular-based vehicular network which we consider in this paper, all the messages are centrally processed by the GeoServer. Such a setting is advantageous for cellular-based vehicular networks, because cellular networks currently do not support multicasting (eMBMS) [7], and large amount of data exchange would be necessary if the GeoServer does not aggregate the information from different vehicles.
} 
as fresh as possible.

The remainder of this paper is organized as follows. In Section II, we outline the related work and highlight the novelty of our work. Section III discusses the framework of the proposed rate control mechanism. Section IV discusses the method of calculating the optimal message rate, based on observations on the network performance. Simulation results are presented in Section V, and Section VI draws conclusions.

\section{Related Work And Problem Statement}

Some existing works on congestion control for multi-hop vehicular networks focus on adjusting the broadcast area, either by varying the number of forwarders [9] or controlling the transmission power of nodes [10], [11]. Ref. [12] makes use of low layer data and adjusts the beacon rate according to the channel busy time. These approaches are infeasible for cellular-based vehicular networks where vehicles do not communicate in a multi-hop fashion, and it is also impractical to obtain low layer data from commercial networks. It follows that a transport layer or application layer congestion control mechanism is desirable for cellular-based vehicular networks.

However, existing high layer congestion control algorithms are not specifically designed for vehicular network applications. Most existing schemes, such as those used in the Transport Control Protocol (TCP), adjust the packet transmission rate based on the packet loss or round-trip time [13]. The rate for each single flow is determined and adjusted independently. Acknowledgment (ACK) packets need to be sent for retransmission purposes as well as for measuring the round-trip time. In cellular-based vehicular networks, we have the following features that allow us to design a more efficient rate control algorithm:

1) All vehicles communicate with the same entity - the GeoServer. The bandwidth is also shared by all the vehicles that are in the same cell (which corresponds to one base-station). Hence, rather than considering rate adjustment for each single flow, we can specify an identical packet rate for all the vehicles in a particular cell (but rates for uplink and downlink may be different). We ensure the fairness ${ }^{2}$ among vehicles in this way, and the rate control mechanism can also make use of packet transmission statistics of multiple vehicles, which can bring faster and more accurate rate adjustments.

2) For vehicular applications, vehicles have to be equipped with Global Positioning System (GPS) devices to measure its speed, position, etc. GPS devices also allow us to obtain precise timing, and it is also not costly to connect a GPS device to the GeoServer. In the presence of a synchronized time, we can measure the delay of packets directly at the receiver side, and use such measurements for packet rate adaptation.

\footnotetext{
${ }^{2}$ Note that we do not consider the impact of packet loss in this paper, because we recognize that the adaptive coding and modulation techniques used in the cellular network would result in similar packet loss rates for different vehicles.
}

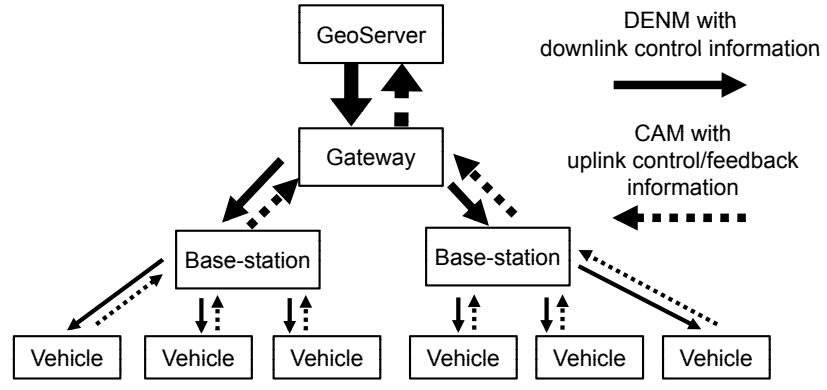

Fig. 2. Information exchange process.

3) The rate control and feedback information can be included in the CAMs and DENMs because they are sent regularly. Hence, no additional control packet is needed.

In this paper, we propose a message rate control mechanism that takes into account the above features.

\section{FRAMEWORK OF RATE CONTROL MEChanism}

The proposed message rate control mechanism utilizes information that is acquirable at the application level. To assist rate adaptation, control and feedback information is added into the actual CAMs and DENMs, and sent via the uplink and downlink channels, as shown in Fig. 2.

In the proposed rate control mechanism, all optimal packet rate calculations are performed at the GeoServer, hence it is centralized. Such a design is with the consideration that the GeoServer coordinates all the vehicles that it is responsible for, which would reduce communication overhead compared with schemes where vehicles coordinate with each other distributively. It also reduces the complexity of the communication device on the vehicle, which is often an embedded system.

The vehicles and the GeoServer collect information from the packets they receive. Such information include: 1) packet delay; 2) packet transmission interval (the reciprocal value of packet rate) at the time when the packet was sent, which is used as a reference for rate optimization because it can be relevant with the packet delay. Due to the centralized setup, data collected at vehicles are fed back to the GeoServer for processing. In this paper, the GeoServer only stores the delay and transmission interval of the latest packet corresponding to the each of the uplink and downlink of each vehicle. Data that correspond to the uplink (or, correspondingly, downlink) of vehicles in the same cell are jointly processed and an identical packet rate is set for the uplink (or, correspondingly, downlink) transmission of these vehicles. In the remaining discussion, we only focus on vehicles in the same cell.

\section{A. Definitions}

In the following notations, we use superscript "Server" to denote that the variable is stored in the GeoServer, and use superscript "Vehicle $i$ " to denote that the variable is stored in vehicle $i$. Let $\mathcal{T}_{\mathrm{UL}}^{\text {Server }}=\left\{\left(T_{\mathrm{d} \text { (latest)-UL }}^{\text {Server }}(i), T_{\text {int(latest)-UL }}^{\text {Server }}(i)\right): \forall i \in\right.$ $\mathcal{V}\}$ and $\mathcal{T}_{\mathrm{DL}}^{\text {Server }}=\left\{\left(T_{\mathrm{d} \text { (latest)-DL }}^{\text {Server }}(i), T_{\text {int(latest)-DL }}^{\text {Server }}(i)\right): \forall i \in \mathcal{V}\right\}$ respectively denote the data regarding uplink and downlink packets that are stored in the GeoServer, where $T_{\mathrm{d}(\text { latest) }}$ and $T_{\text {int(latest) }}$ are respectively the delay and packet interval of the 
latest packet, "UL" or "DL" in the subscript denotes whether uplink or downlink is considered, $\mathcal{V}$ is the set of indexes of all vehicles, and the argument $i$ denotes the vehicle index. The optimal uplink packet interval $T_{\text {int(opt)-UL }}^{\text {Server }}\left(\mathcal{T}_{\mathrm{UL}}^{\text {Server }}\right)$ (or, correspondingly, downlink packet interval $\left.T_{\text {int(opt)-DL }}^{\text {Server }}\left(\mathcal{D}_{\mathrm{DL}}^{\text {Server }}\right)\right)$ is evaluated based on data in $\mathcal{T}_{\mathrm{UL}}^{\text {Server }}$ (or, correspondingly,

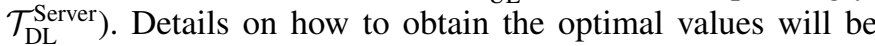
discussed in Section IV. In addition, the GeoServer maintains a time $t_{\mathrm{DL}}^{\text {Server }}(i)$ for each vehicle $i$, which is the time when the next packet to vehicle $i$ will be sent. It also maintains a time $t_{\text {prev-DL }}^{\text {Server }}(i)$, which is the time when the previous packet was sent to vehicle $i$. At the vehicle side, each vehicle $i$ maintains $T_{\text {int(opt)-UL }}^{\text {Vehicl } i}$ which stores the current (optimal) uplink packet interval specified by the GeoServer. Upon receiving a packet from the GeoServer, vehicle $i$ stores the delay and the transmission interval of the packet respectively into $T_{\mathrm{d}(\text { latest)-DL }}^{\text {Vecicle } i}$ and $T_{\text {int(latest)-DL }}^{\text {Vehicle } i}$. Also, each vehicle $i$ maintains a time $t_{\mathrm{UL}}^{\text {Vehicle } i}$, which is the time when the next packet will be sent to the GeoServer; and also $t_{\text {prev-UL }}^{\text {Vehicle } i}$, which is the time when the previous packet was sent to the GeoServer. We denote the current time by $t_{\text {now }}$.

The downlink and uplink packets that the GeoServer and vehicles respectively generate are respectively denoted by vectors $p_{\mathrm{DL}}=\left(t_{\mathrm{now}}, T_{\text {int(opt)-UL }}^{\text {Server }}\left(\mathcal{T}_{\mathrm{UL}}^{\text {Server }}\right), T_{\text {int(opt)-DL }}^{\text {Server }}\left(\mathcal{T}_{\mathrm{DL}}^{\text {Server }}\right)\right)$ and $p_{\mathrm{UL}}=\left(t_{\mathrm{now}}, T_{\text {int(opt)-UL }}^{\mathrm{Vehicle} i}, T_{\mathrm{d} \text { (latest)-DL }}^{\mathrm{Vehicle} i}, T_{\text {int(latest)-DL }}^{\mathrm{Vehicle} i}\right)$, which contain essential information for rate adaptation. Other information for downlink packets can include the index of the vehicle to which the packet is being sent to, a sequence number, and the actual DENM content; other information for uplink packets can include the index of the cell that the vehicle is currently in, a sequence number, and the actual CAM content. Such information has been omitted in $p_{\mathrm{DL}}$ and $p_{\mathrm{UL}}$ for the ease of expression.

\section{B. Rate Adjustment and Packet Scheduling Procedures}

The packet interval adjustment procedure is combined into the packet scheduling and transmission procedure. The procedures performed at the GeoServer and each vehicle are respectively shown in Algorithms 1 and 2.

As illustrated in Algorithm 1, upon receiving a packet from any vehicle $i$ (i.e. when the condition in line 3 is satisfied), the GeoServer computes the delay of this packet and updates $\mathcal{T}_{\mathrm{UL}}^{\text {Server }}$ and $\mathcal{T}_{\mathrm{DL}}^{\text {Server }}$ with the new information from vehicle $i$ (lines 4-8). Afterwards, it recomputes the optimal downlink interval $T_{\text {int(opt)-DL }}^{\text {Serve }}$ and reschedules the next packet transmission for all vehicles with reference to the time when the previous packet was sent out to a particular vehicle (lines 9-11). When the scheduled time for a packet transmission to a particular vehicle $i$ has approached (i.e. the condition in line 13 is satisfied), the GeoServer generates a downlink packet with the the optimal uplink and downlink intervals $T_{\text {int(opt)-UL }}^{\text {Serer }}$ and $T_{\text {int(opt)-DL }}^{\text {Server }}$ that are respectively computed based on the current sets of $\mathcal{T}_{\mathrm{UL}}^{\text {Server }}$ and $\mathcal{T}_{\mathrm{DL}}^{\text {Server }}$ (lines 14-15), and the packet is sent to vehicle $i$ (line 16). Afterwards, the next packet transmission is scheduled according to $T_{\text {int(opt)-DL }}^{\text {Server }}\left(\mathcal{T}_{\mathrm{DL}}^{\text {Server }}\right)$ (lines 17-18).

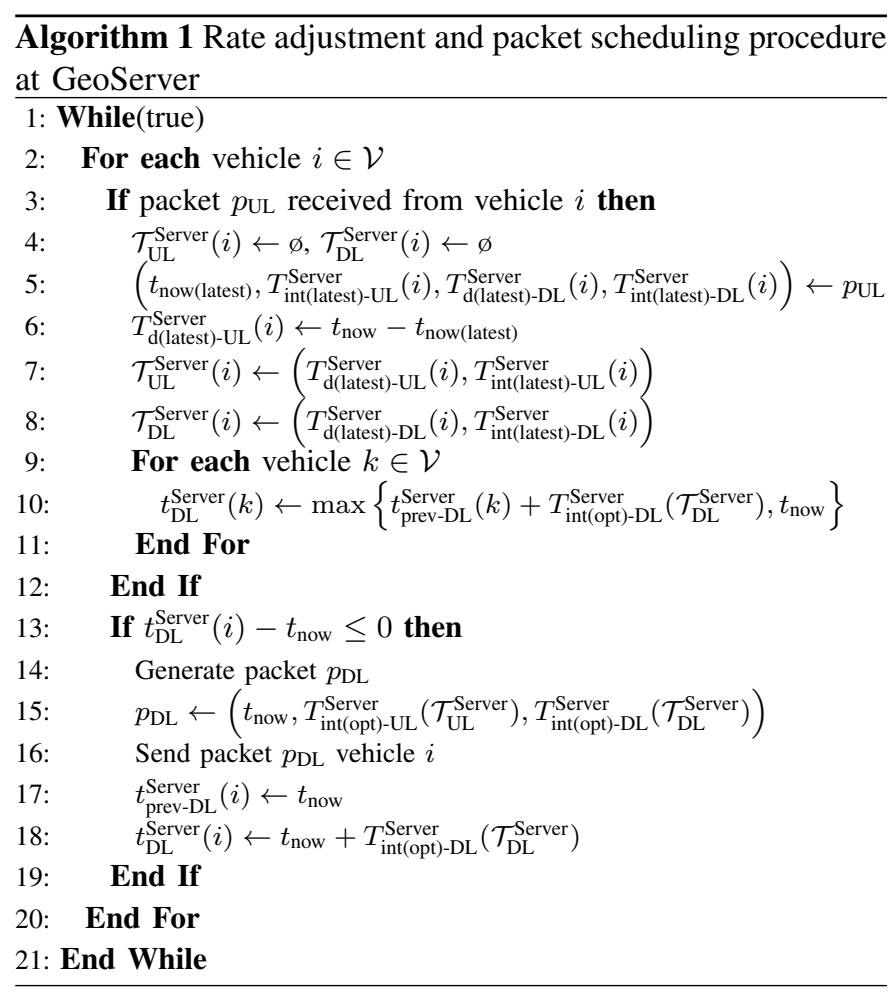

The procedure at vehicles is similar with that at the GeoServer, except that vehicles do not compute optimal packet intervals by themselves but use the intervals specified by the GeoServer (and sent to vehicles in downlink packets), as illustrated in Algorithm 2. When transmitting a packet to the GeoServer, the measured delay and the transmission interval of the latest downlink packet are included into the (uplink) packet for feedback.

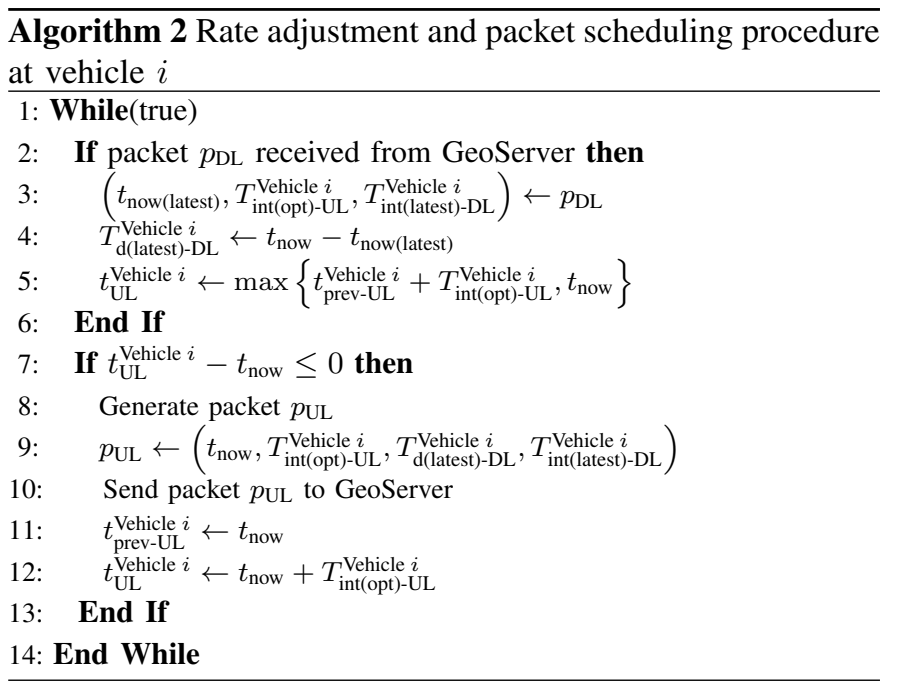

Although we use the "While" statement in Algorithms 1 and 2, the system may operate in an event-based mechanism in practice, where packet reception and packet transmission are discrete-time events. 


\section{Determining the Optimal Packet Interval}

In this section, we discuss how the GeoServer obtains the optimal uplink and downlink packet intervals $T_{\text {int(opt)-UL }}^{\text {Server }}$ and $T_{\text {int (opt)-DL }}^{\text {Server }}$, based on data in $\mathcal{T}_{\mathrm{UL}}^{\text {Server }}$ and $\mathcal{T}_{\mathrm{DL}}^{\text {Server }}$. Vehicles in different cells and/or with different transmission directions (i.e. either uplink or downlink) are individually considered, following the same procedure. Hence, in this section, we only focus on vehicles in the same cell and with the same transmission direction, and omit the subscripts "UL" and "DL". We also omit the superscript "Server" because all rate calculations are performed at the GeoServer. With such notations, we would like to find $T_{\text {int(opt) }}(\mathcal{T})$, where $\mathcal{T}=\left\{\left(T_{\text {d(latest) }}(i), T_{\text {int(latest })}(i)\right)\right.$ : $\forall i \in \mathcal{V}\}$ is the set of stored delay and transmission intervals of the latest packets corresponding to all vehicles for either uplink or downlink, and the resulting $T_{\text {int(opt) }}$ is the optimal interval for either uplink or downlink packets depending on which set of data has been considered.

\section{A. Problem Formulation}

Because most vehicular network applications are timecritical, the goal of the rate adjustment is to maintain the information at the vehicles and the GeoServer as new as possible. Toward this goal, we first define a metric to measure the newness or freshness of the information that is stored at the vehicle or the GeoServer. We name this metric as information depreciation, and it is defined as the maximum difference between the current time and the time when the information has been gathered at the vehicle (or, correspondingly, the GeoServer) and sent out to the GeoServer (or, correspondingly, the vehicle). Mathematically, the information depreciation for vehicle $i$ (denoted by $D(i)$ ) is defined as follows:

$$
D(i)=T_{\text {int }}(i)+T_{\mathrm{d}}(i),
$$

where $T_{\text {int }}(i)$ is the interval and $T_{\mathrm{d}}(i)$ is the total delay (including queuing and transmission delay) of the packet corresponding to vehicle $i$, and $T_{\mathrm{d}}(i)$ is dependent on $T_{\mathrm{int}}(i)$.

The goal of the rate adjustment is to find an optimal packet interval $T_{\text {int(opt) }}$ that minimizes the average information depreciation of all vehicles, i.e. we would like to find

$$
T_{\text {int(opt) }}=\arg \min _{\overline{T_{\text {int }}}} \bar{D}=\arg \min _{\overline{T_{\text {int }}}}\left(\overline{T_{\text {int }}}+\overline{T_{\mathrm{d}}}\right),
$$

where $\bar{X}=\frac{1}{|\mathcal{V}|} \sum_{i \in \mathcal{V}} X(i)$ denotes the average value over all vehicles in set $\mathcal{V}, X$ can be an arbitrary variable that is related with the vehicle index $i$, and $|\mathcal{V}|$ is the number of elements in $\mathcal{V}$. The packet interval $T_{\text {int }}$ may also vary with $i$ because, although we attempt to set the packet interval of all vehicles to the same value, the optimal rate may change over time due to newly collected samples.

\section{B. Optimal Solution}

To evaluate the value of $\overline{T_{\mathrm{d}}}$ in (2), we consider a queuing model as shown in Fig. 3. We abstract the uplink and downlink of each vehicle as independent queues which store the packets for transmission. This abstraction is with the consideration that cellular networks generally operate with a sharing policy among users, where each user has independent queues for

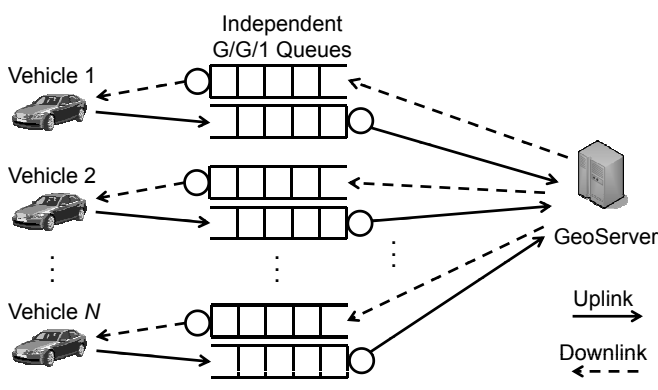

Fig. 3. Queuing model.

both uplink and downlink. We also assume that the queues of the vehicles in the same cell have identical service time distribution $^{3}$ as long as the same transmission direction (i.e. either uplink or downlink) is considered. This is due to the fact that the total bandwidth is shared among users with some fairness-aware scheduling policy, thus the necessary transmission time (which corresponds to the service time) of packets from different users have some statistical similarities. The service time can also capture the impact of other traffics in the network, and a higher amount of cross-traffic may cause a larger service time. Hence, we do not specifically consider the cross-traffic in our analysis.

Because we expect to obtain more accurate results when the traffic load is high (but the network should still be uncongested), we use the heavy traffic approximation [14], and the total delay can be approximated by

$$
\overline{T_{\mathrm{d}}} \approx \frac{\sigma_{T_{\mathrm{int}}}^{2}+\sigma_{S}^{2}}{2\left(\overline{T_{\mathrm{int}}}-\bar{S}\right)}+\bar{S},
$$

where $S(i)$ is the service time for vehicle $i$, and $\sigma_{X}^{2}=$ $\frac{1}{|\mathcal{V}|} \sum_{i \in \mathcal{V}}(X(i)-\bar{X})^{2}$ denotes the variance, $X$ can be an arbitrary variable that is related with the vehicle index $i$.

Substituting (3) into the objective function in (2), we have

$T_{\mathrm{int}(\mathrm{opt})}=\arg \min _{\overline{\overline{\mathrm{int}}_{\mathrm{int}}}} \bar{D}=\arg \min _{\overline{T_{\mathrm{int}}}}\left(\overline{T_{\mathrm{int}}}+\frac{\sigma_{T_{\mathrm{int}}}^{2}+\sigma_{S}^{2}}{2\left(\overline{T_{\mathrm{int}}}-\bar{S}\right)}+\bar{S}\right)$.

Note that the packet interval must be larger than $\bar{S}$ so that the queue remains stable, solving for $\left.\frac{\partial \bar{D}}{\partial \bar{T}_{\text {int }}}\right|_{T_{\text {int }}=T_{\text {int(opt) }}}=0$, we obtain the optimal packet interval as

$$
T_{\text {int(opt) }}=\bar{S}+\frac{\sqrt{2\left(\sigma_{T_{\text {int }}^{2}}^{2}+\sigma_{S}^{2}\right)}}{2} .
$$

Eq. (5) results in the minimum average depreciation, because

$$
\left.\frac{\partial^{2} \bar{D}}{\partial{\overline{\overline{\mathrm{int}}^{2}}}^{2}}\right|_{\overline{T_{\mathrm{int}}}=T_{\mathrm{in}(\mathrm{opt})}}=\frac{\sigma_{T_{\mathrm{int}}}^{2}+\sigma_{S}^{2}}{\left(T_{\mathrm{int}(\mathrm{opt})}-\bar{S}\right)^{3}}>0 .
$$

\section{Estimating the Parameters}

To evaluate the optimal packet interval from (5), we need to know $\sigma_{T_{\mathrm{int}}}^{2}, \bar{S}$, and $\sigma_{S}^{2}$. We estimate these values from the data

\footnotetext{
${ }^{3}$ Note that the individual service times may be different, but we assume they follow the same distribution.
} 
stored in $\mathcal{T}$. From $\left(T_{\text {d(latest) }}(i), T_{\text {int(latest })}(i)\right) \in \mathcal{T}, \forall i \in \mathcal{V}$, we can directly obtain the values of $\overline{T_{\text {int(latest })}}, \sigma_{T_{\text {int(latest }}^{2}}, \overline{T_{\mathrm{d}(\text { latest })}}$, and $\sigma_{T_{\text {d(atatest }}}^{2}$. We can consider the samples of all vehicles because we assume identical packet interval distribution and identical service time distribution among all vehicles, as aforementioned. Then, the estimation of $\sigma_{T_{\mathrm{int}}}^{2}$ is straightforward with

$$
\sigma_{T_{\text {int }}}^{2}=\sigma_{T_{\text {int(latest }}^{2}}^{2} .
$$

By letting $\overline{T_{\text {int }}}=\overline{T_{\text {int(latest) }}}, \sigma_{T_{\text {int }}}^{2}=\sigma_{T_{\text {int(latest) }}^{2}}$, and $\overline{T_{\mathrm{d}}}=\overline{T_{\mathrm{d}(\text { latest })}}$ in (3), and also considering that $\overline{T_{\text {int(latest) }}}>\bar{S}$ so that the queue remains stable, we have $\overline{T_{\mathrm{d}(\text { latest })}} \geq \bar{S}$, and we can solve for $\bar{S}$ from (3) as

$$
\bar{S}=\frac{\overline{T_{\text {int(latest })}}+\overline{T_{\text {d(latest })}}-\sqrt{F}}{2},
$$

where $F \triangleq\left(\overline{T_{\text {int(latest })}}-\overline{T_{\mathrm{d}(\text { latest })}}\right)^{2}+2\left(\sigma_{T_{\text {int(latest })}^{2}}+\sigma_{S}^{2}\right)$.

To estimate the value of $\sigma_{S}^{2}$, we note that the delay at vehicle $i$ is $T_{\mathrm{d}(\text { latest })}(i)=\sum_{j=1}^{N(i)} S_{j}(i)+R(i)$, where $N(i)$ is the number of packets in the queue, $S_{j}(i)$ is the service time of the $j$ th packet in the queue, and $R(i)$ is the residual service time of the packet that is being transmitted, all for vehicle $i$. Variables $N(i)$ and $R(i)$ are captured when the packet under consideration enters the queue. We assume that $S_{j}(i)$ for different $j$ are independent, and they are also independent of $R(i)$, because they generally belong to different scheduling rounds. With an approximation of $\sigma_{S}^{2} \approx \sigma_{R}^{2}$, and also due to the heavy traffic consideration so that we assume that there is (almost) always a packet in service, we have $\sigma_{T_{\text {d(latest }}}^{2} \approx(\bar{N}+1) \sigma_{S}^{2}$. The value of $\bar{N}+1$ can be approximated by the average number of packet arrivals during the packet delay time, i.e. $\bar{N}+1 \approx \max \left\{\frac{\overline{\mathrm{d}}_{\mathrm{d}(\mathrm{atats})}}{\overline{T_{\text {int(latest }}}}, 1\right\}$. Then, we have

$$
\sigma_{S}^{2} \approx \sigma_{T_{\mathrm{d}(\text { latess) }}}^{2} \cdot \min \left\{\frac{\overline{T_{\text {int(latest) }}}}{\overline{T_{\mathrm{d}(\text { latest })}}}, 1\right\} .
$$

\section{When Assumptions Do Not Hold}

Practical systems may deviate from the assumptions and approximations made in the previous analysis. In particular, (3) only holds when the network is not congested. When the assumptions do not exactly hold, the proposed scheme can still provide negative feedback which prevents the network from being congested. This follows from the fact that, substituting (7) and (8) into (5), we have

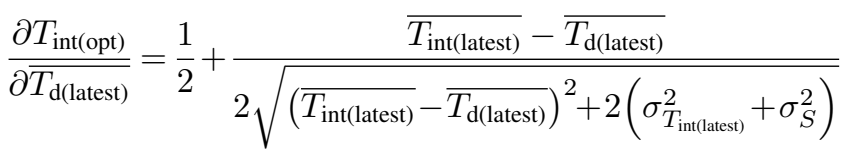$$
\geqslant 0
$$

with equal sign if and only if $\sigma_{T_{\text {int(latest) }}^{2}}=\sigma_{S}^{2}=0$ (which is very

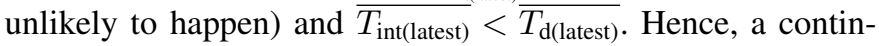
uously increasing delay that may be due to congestion would also lead to larger packet interval which would subsequently relief the congestion.

Additionally, to allow fast recovery from network congestion, we define a congestion avoidance factor $\alpha$ and compare the values of $\overline{T_{\mathrm{d}(\text { latest })}}$ and $\overline{T_{\text {int(latest) }}}$. If $\overline{T_{\mathrm{d}(\text { latest })}}>\alpha \overline{T_{\text {int(latest })}}$, we set the optimal interval directly to the average delay, i.e. $T_{\text {int(opt })}=\overline{T_{\mathrm{d}(\text { latest })}}$, so that the number of packets that are backlogged in the network does not increase. After the network is no longer congested (we judge this by $\overline{T_{\mathrm{d}(\text { latest })}} \leq \alpha \overline{T_{\text {int(latest) }}}$ ), we continue with the normal operation.

\section{E. Procedure}

According to the above discussions, the procedure of the optimal packet interval calculation can be summarized as Algorithm 3.

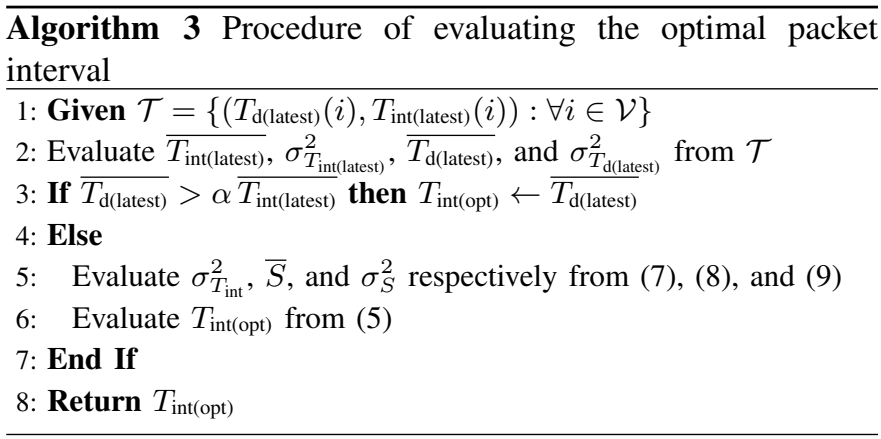

\section{Simulation Results}

We evaluate the performance of the proposed rate control mechanism via simulations in the network simulator NS-3 [15]. An LTE network with $5 \times 5 \mathrm{MHz}$ uplink/downlink bandwidth with one base-station (eNodeB in the LTE network) and multiple number of vehicles is considered. The network area is $2000 \mathrm{~m} \times 2000 \mathrm{~m}$ where the eNodeB is placed in the center and the vehicles are randomly distributed within this area. We focus on the scenario with one eNodeB due to simulation efficiency considerations. The results with multiple base-stations would be similar because we treat each cell independently. The transmission power of each vehicle that corresponds to the user equipment (UE) in the LTE network is set to $10 \mathrm{dBm}$, and the transmission power of the eNodeB is $30 \mathrm{dBm}$. A round robin scheduler is used in the network. We consider two different mobility and fading scenarios in the simulations. In the first scenario, the vehicles do not move and the wireless channel does not exhibit fading, which is for evaluating the performance in the most ideal case. In the second scenario, the vehicles randomly move at $16.67 \mathrm{~m} / \mathrm{s}(60$ $\mathrm{km} / \mathrm{h}$ ) and we use a fading trace obtained from the Extended Vehicular A (EVA) model as defined in [16], to represent more realistic cases. The CAM (uplink) packet size is 90 Bytes and the DENM (downlink) packet size is uniformly distributed within 170 and 270 Bytes. The initial packet interval is set to $3 \mathrm{~s}$, which is a relatively large value to avoid network congestion. The congestion avoidance factor $\alpha$ is set to 2 . We run the simulation for $100 \mathrm{~s}$ and with nine different random seeds to obtain the overall performance.

\section{A. Adapted Packet Intervals}

We first consider the packet intervals that are obtained from the proposed mechanism. In Fig. 4(a), we plot the instant optimal packet intervals against time when running 


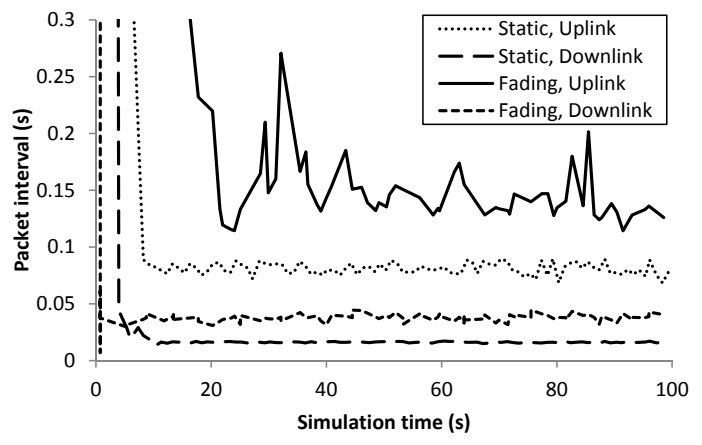

(a)

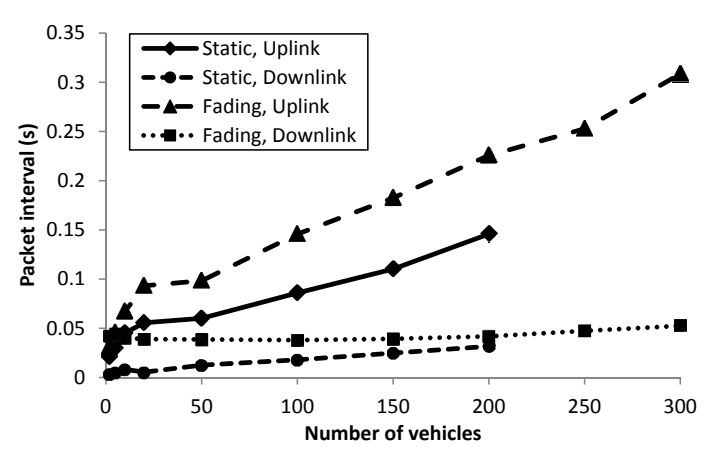

(b)

Fig. 4. Adapted packet intervals: (a) instant intervals when the number of vehicles is 100, (b) average intervals.

the simulation with 100 vehicles and a particular random seed. We can observe that except for the uplink in the fading scenario, the packet intervals converge to relatively stable values within $10 \mathrm{~s}$ of network initialization. The fluctuation is small after the stable value has been reached. The uplink in the fading scenario exhibits some larger fluctuations because the transmission power of the vehicles is lower than that of the base-station, which means that the uplink is more sensitive to the random fading status leading to a larger fluctuation.

Fig. 4(b) shows the average packet intervals that are collected after $20 \mathrm{~s}$ of simulation time. As expected, the packet intervals increase with the number of vehicles, to avoid congestion. Also, the packet intervals in the fading scenario is larger than that in the static scenario, because the randomness of fading can cause lower data transmission rate of the LTE network to maintain the bit-error rate below a certain level.

\section{B. Average Depreciation}

To study the performance of information depreciation when using the proposed mechanism, we compare the results with the fixed rate scheme as in [7]. For a fair comparison, the packet intervals of the fixed rate scheme are set to the corresponding optimal interval that we obtain from the proposed scheme when the number of vehicles is 100 . The average depreciation is collected after $20 \mathrm{~s}$ of simulation time. Results are shown in Figs. 5 and 6. The relative reduction of depreciation is defined as $-\left(D_{\text {proposed }}-D_{\text {fixed }}\right) / D_{\text {fixed }}$, where $D_{\text {proposed }}$ and $D_{\text {fixed }}$ respectively denote the depreciations when using the proposed and fixed rate schemes.

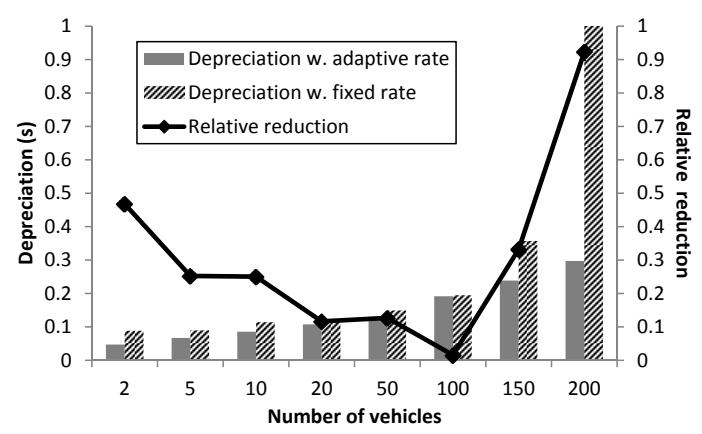

(a)

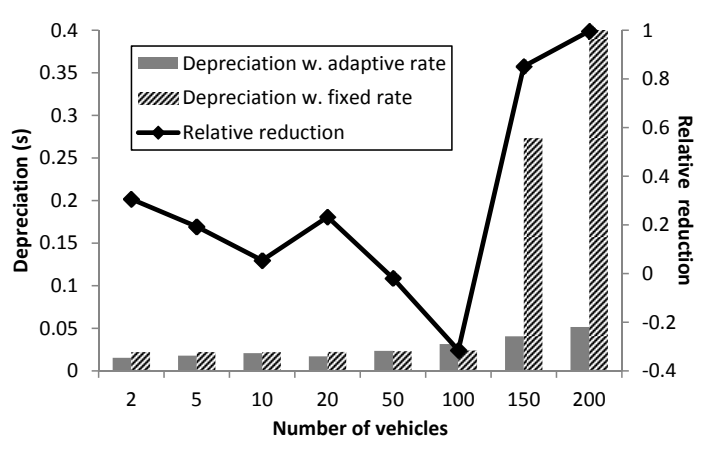

(b)

Fig. 5. Average depreciation in the static scenario: (a) uplink, (b) downlink.

In the static scenario, we can observe from Fig. 5 that the proposed adaptive rate mechanism can always bring lower or equal depreciation for the uplink. For the downlink, when there are 100 vehicles, the proposed mechanism brings slightly higher depreciation compared with the fixed rate method. The reason is, as shown in Fig. 4(a), the packet interval variation in this case is very low, and its value is also small. Hence, according to (5), the packet interval is set close to $\bar{S}$, and a very small deviation of the packet interval may cause the network to operate in the congested status. Therefore, the proposed scheme provides slightly worse performance compared with the fixed rate scheme that exactly and always uses the optimal interval when there are 100 vehicles. However, such a performance degradation is not significant when noticing the depreciation itself is very low, and it is infeasible to always use the exact optimal rate in practice. The fact that the depreciation is lowest when there are 100 vehicles also confirms that the optimal packet intervals resulting from the proposed mechanism is close to the true optimum.

In the fading scenario, the proposed scheme is generally beneficial as shown in Fig. 6. The fact that the proposed method performs slightly worse than the fixed rate scheme in the uplink when there are around 150 or 200 vehicles is due to the randomness of fading and its impact on the uplink. Such randomness increases the variance of the packet delay. Because we compute the packet interval according to (5), the increased variance makes the packet interval value higher than the actual optimum. However, it is very difficult to judge at the application level whether the randomness comes from fading or queuing. In the downlink, the performance is slightly worse 


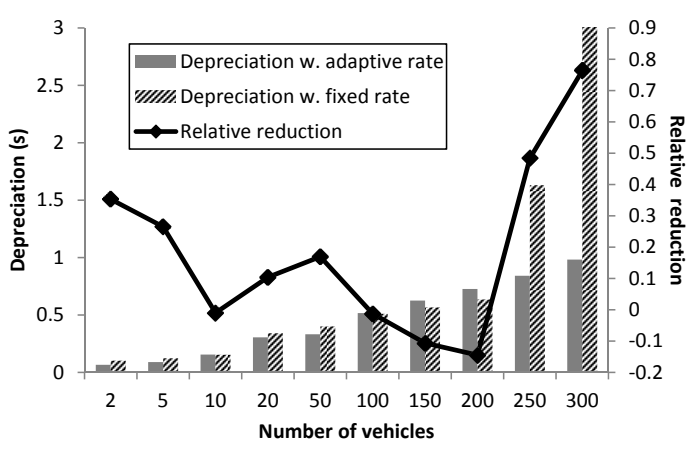

(a)

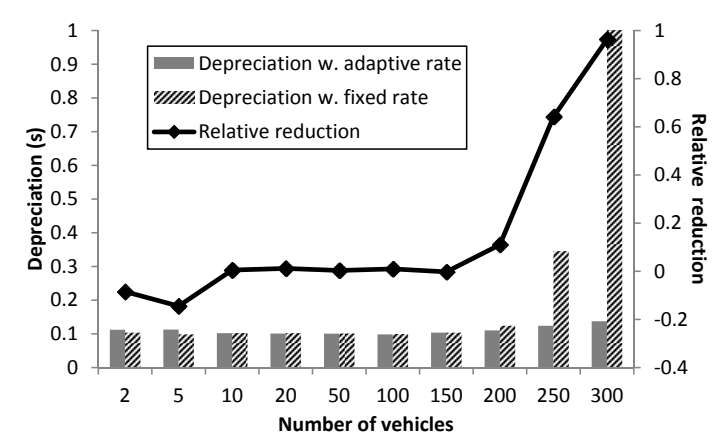

(b)

Fig. 6. Average depreciation in the fading scenario: (a) uplink, (b) downlink.

when there are few vehicles, which is mainly because the number of samples is limited in this case, leading to some estimation inaccuracies.

Overall, the results show that the proposed mechanism can generally provide advantageous depreciation performance. It also successfully avoids network congestion and has low packet interval fluctuation. The relative depreciation reduction ranges from -0.3 to 1.0 compared with the fixed rate scheme.

\section{CONCLUSION}

In this paper, we have proposed a rate adaptation scheme for cellular-based vehicular networks, which adjusts the network load to keep the information as new as possible, while concurrently avoiding network congestion. The proposed mechanism takes into account the features of vehicular networking, which enable us to perform relatively accurate rate control without the need of much additional signaling overhead. Further, its goal is to minimize information depreciation, and information newness can be regarded as one of the most significant factors for vehicular networks. The rate adjustment is based on information that can be obtained at the application level. It can work with the User Diagram Protocol (UDP) stack and no additional modification to the protocol stack is necessary. The optimal packet rate is directly evaluated from a mathematical formula, which is easy to implement in practical systems. In this paper, only the delay and transmission rate of the latest received packet is used for calculation. However, the framework that we introduce allows the incorporation of other network performance parameters (e.g. packet loss) or context information (e.g. speed and position of vehicles) in the future. The inclusion of such information may also allow us to estimate the fading status of vehicles and enhance the performance of the proposed scheme in fading scenarios. Although we have mainly focused on the single cell case in this paper, the framework can be extended to multiple cells. When vehicles handover between cells, historical information for other vehicles may be copied for the newly entered vehicle, because vehicles in the same area generally exhibit similar performance.

\section{ACKNOWLEDGMENT}

This work was performed primarily at NEC Laboratories Europe, NEC Europe Ltd., Heidelberg, Germany where the first author worked as an intern employee in 2012.

\section{REFERENCES}

[1] ETSI Technical Committee Intelligent Transport System, Intelligent Transport Systems (ITS); Vehicular Communications; Basic Set of Applications; Part 1: Functional Requirements, Technical Specification: ETSI TS 102 637-1 V1.1.1 (2010-09).

[2] E. Hossain, G. Chow, V. C. Leung, R. D. McLeod, J. Misic, V. W Wong, and O. Yang, "Vehicular telematics over heterogeneous wireless networks: A survey," Computer Communications, vol. 33, no. 7, pp. 775-793, 2010.

[3] C. Birle, B. Borsetzky, Y. Chen, U. Dietz, G. Gehlen, S. Glaser, G. Jodlauk, J. Kahle, G. Nocker, A. Schmidt, C. Sommer, and S. Sories, "CoCar feasibility study: Technology, business and dissemination," CoCar Consortium, Tech. Rep., 2009.

[4] L. Le, A. Festag, A. Mader, R. Baldessari, M. Sakata, T. Tsukahara, and M. Kato, "Infrastructure-assisted communication for car-to-X communication," in Proc. 18th ITS World Congress 2011, Oct. 2011, pp. $1-7$.

[5] A. Festag, M. Wiecker, and N. Zahariev, "Safety and traffic efficiency applications for GeoMessaging over cellular mobile networks," in Proc. 19th ITS World Congress 2012, Oct. 2012, pp. 1-8.

[6] ETSI Technical Committee Intelligent Transport System, Intelligent Transport Systems (ITS); Vehicular Communications; Basic Set of Applications; Part 2: Specification of Cooperative Awareness Basic Service, Technical Specification: ETSI TS 102 637-2 V1.2.1 (2011-03).

[7] M.-A. Phan, R. Rembarz, and S. Sories, "A capacity analysis for the transmission of event and cooperative awareness messages in LTE networks," in Proc. 18th ITS World Congress 2011, Oct. 2011, pp. 1-12.

[8] ETSI Technical Committee Intelligent Transport System, Intelligent Transport Systems (ITS); Vehicular Communications; Basic Set of Applications; Part 3: Specifications of Decentralized Environmental Notification Basic Service, Technical Specification: ETSI TS 102 637-3 V1.1.1 (2010-09).

[9] N. Wisitpongphan, O. Tonguz, J. Parikh, P. Mudalige, F. Bai, and V. Sadekar, "Broadcast storm mitigation techniques in vehicular ad hoc networks," IEEE Wireless Communications, vol. 14, no. 6, pp. 84-94, Dec. 2007.

[10] G. Caizzone, P. Giacomazzi, L. Musumeci, and G. Verticale, "A power control algorithm with high channel availability for vehicular ad hoc networks," in Proc. IEEE ICC 2005, vol. 5, May 2005, pp. 3171-3176.

[11] M. Torrent-Moreno, P. Santi, and H. Hartenstein, "Distributed fair transmit power adjustment for vehicular ad hoc networks," in Proc. IEEE SECON 2006, vol. 2, Sep. 2006, pp. 479-488.

[12] L. Le, R. Baldessari, P. Salvador, A. Festag, and W. Zhang, "Performance evaluation of beacon congestion control algorithms for VANETs," in Proc. IEEE GLOBECOM 2011, Dec. 2011, pp. 1-6.

[13] A. Tanenbaum, Computer Networks, Fourth Edition. New Jersey: Prentice Hall, 2003.

[14] L. Kleinrock, Queuing Systems, Volume II: Computer Applications. Hoboken, NJ: John Wiley and Sons, 1976.

[15] Network Simulator 3 (NS-3). [Online]. Available: http://www.nsnam.org/

[16] 3rd Generation Partnership Project (3GPP), 3rd Generation Partnership Project; Technical Specification Group Radio Access Network; Evolved Universal Terrestrial Radio Access (E-UTRA); Base Station (BS) radio transmission and reception (Release 8), Technical Specification: 3GPP TS 36.104 V8.0.0 (2007-12). 